\title{
Theory of optimum shapes in free-surface flows. Part 2. Minimum drag profiles in infinite cavity flow
}

\author{
By ARTHUR K. WHITNEY $\dagger$ \\ California Institute of Technology, Pasadena, California
}

(Received 8 September 1971 and in revised form 6 July 1972)

The problem considered here is to determine the shape of a symmetric twodimensional plate so that the drag of this plate in infinite eavity flow is a minimum. With the flow assumed steady and irrotational, and the effects due to gravity ignored, the drag of the plate is minimized under the constraints that the frontal width and wetted arc-length of the plate are fixed. The extremization process yields, by analogy with the classical Euler differential equation, a pair of coupled nonlinear singular integral equations. Although analytical and numerical attempts to solve these equations prove to be unsuccessful, it is shown that the optimal plate shapes must have blunt noses. This problem is next formulated by a method using finite Fourier series expansions, and optimal shapes are obtained for various ratios of plate arc-length to plate width.

\section{Introduction}

We consider the two-dimensional cavity flow of an incompressible fluid past a symmetric plate of arbitrary shape. The flow far upstream is uniform with velocity $U$, pressure $p_{\infty}$, and density $\rho$. The pressure $p_{c}$ inside the cavity is assumed to be a constant, so that by Bernoulli's law the fluid velocity at the cavity wall is a constant $V$, where

$$
\frac{1}{2} \rho V^{2}+p_{c}=\frac{1}{2} \rho U^{2}+p_{\infty} .
$$

The cavity flow may be characterized by the cavitation number

$$
\sigma=\left(p_{\infty}-p_{c}\right) / \frac{1}{2} \rho U^{2}
$$

As the cavitation number decreases, the length and width of the cavity grow indefinitely and the flow approaches 'Helmholtz flow', in which the cavity is infinitely long and the cavity pressure equals the free-stream pressure $(\sigma=0)$.

The specific problem considered here is to find the shape of a symmetric plate (see figure 1) of given wetted are-length $s_{0}$ and given frontal width $y_{0}$, so that the drag of this plate in infinite cavity flow $(\sigma=0)$ is a minimum. A precise definition of the class of plates under consideration will be given in $\S 2$. The solution of this problem has obvious applications in the design of struts or other two-dimensional non-lifting surfaces which may operate in the super-cavitating range. For flows

$\dagger$ Present address: Lockheed Palo Alto Research Laboratory, Lockheed Missiles and Space Co., Palo Alto, California. 


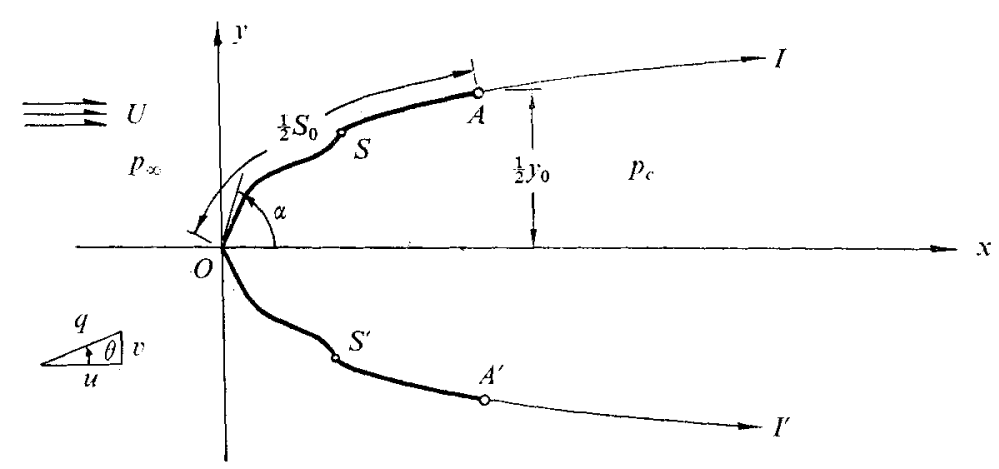

Figure 1. The physical plane. Note that $S A$ and $S^{\prime} A^{\prime}$ are parts of the body boundary that are exposed to the same constant pressure $p_{c}$ as on the cavity boundary.

at large Reynolds number, the viscous effects may be ignored as a first approximation; however, corrections due to viscous drag can be calculated once the potential flow is known. Although optimal shapes are sought for the case $\sigma=0$, these shapes should remain approximately optimal for $\sigma>0$, since the rule

$$
C_{D}(\sigma)=C_{D}(0)(1+\sigma)
$$

is known to relate approximately the drag coefficient $C_{D}$ at $\sigma=0$ to the drag coefficient of the same body for $0<\sigma \leqslant 1$ (see e.g. Gilbarg 1960). Thus, to minimize $C_{D}$ at a given $\sigma>0$, we could just as well minimize $C_{D}(0)$. It should be noted that this rule appears to be a good approximation only for blunt bodies, so the above argument may be less accurate when the arc-length becomes much larger than the width of the plate.

Lavrentieff (1938) gave the solution to a related minimum drag problem (see e.g. Gilbarg 1960), under the constraining condition that the plate is confined to lie within a rectangle which circumscribes the nose and ends of the plate. If the nose of the plate is at the origin and the plate ends are at $\left(x_{0}, \pm \frac{1}{2} y_{0}\right)$, the solution for the optimal profile (see figure 2) was found by Lavrentieff to consist of a flat nose section, of breadth $h$ and the free streamlines ensuing from the ends of this section, as a continuation of the body, to pass through $\left(x_{0}, \pm \frac{1}{2} y_{0}\right)$, the flat nose length $h$, being uniquely determined for given $x_{0}$ and $y_{0}$. The pressure difference across the flat portion of the plate is the only contribution to drag, since the fluid pressure equals the cavity pressure $\left(p=p_{c}\right)$ on the free streamlined sections of the plate. This solution was obtained by the use of several comparison and monotonicity theorems which follow from the maximum principle for harmonic functions.

The present work was originally conceived as an extension to Lavrentieff's problem; but it was hoped that the general problem with varied constraining conditions could be solved by using the variational calculus method introduced earlier by Wu \& Whitney (1971). It may be noted that if the condition that the plate be confined to lie within the rectangle is dropped, one can easily construct a sequence of plate shapes which, in the limit, have zero drag (neglecting viscous 


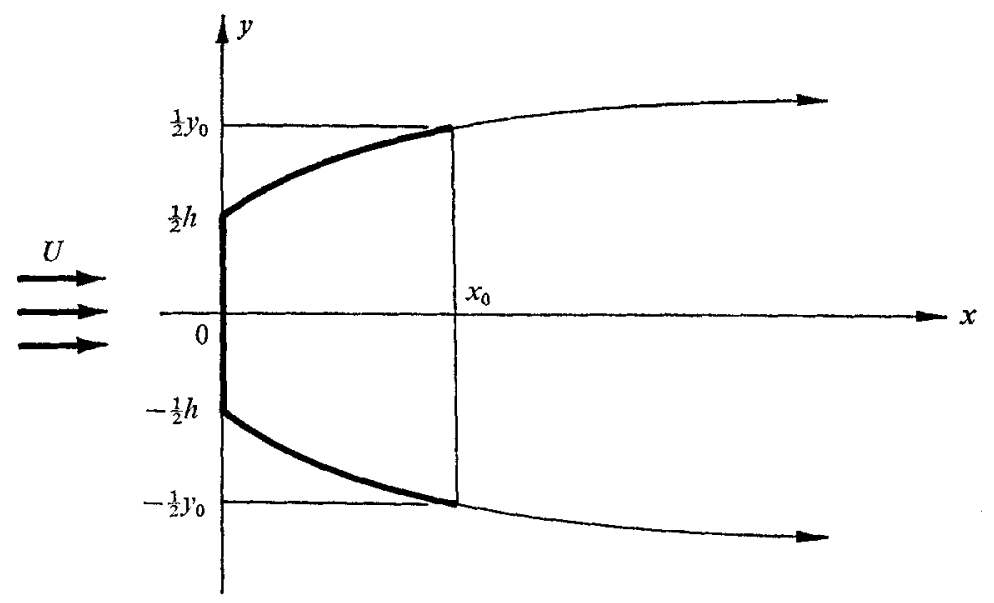

Figure 2. The Lavrentieff profile.

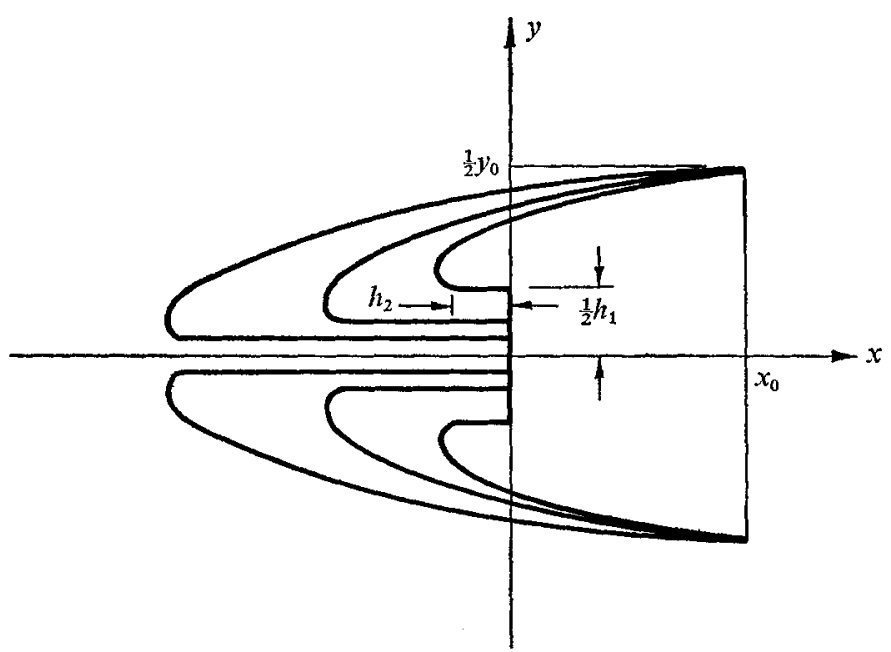

Figure 3. A sequence of plate shapes tending towards one with vanishing drag.

effects). Such a sequence is illustrated in figure 3. A typical plate consists of an inverted cap of width $h_{1}$ and length $h_{2}$ plus the free streamlines which issue from the ends of the cap and go on to pass through the corners of the rectangle. All other plate shapes in this sequence are found by decreasing $h_{1}$ and increasing $h_{2}$ so that the free streamlines always pass through $\left(x_{0}, \pm \frac{1}{2} y_{0}\right)$. As $h_{1} \rightarrow 0$, the flow inside the cap becomes a dead water region with stagnation pressure

$$
p_{s}=\frac{1}{2} \rho U^{2}+p_{\infty},
$$

so that the drag of the plate is just $\frac{1}{2} \rho U^{2} h_{1}$, which can be made arbitrarily small by proper choice of $h_{1}$. Note that the pressure difference across the back face of the cap is the only contribution to drag.

This observation led the author to consider the problem described earlier. It was thought that by fixing the arc-length of the plate, shapes such as those described above (with vanishing drag) would be eliminated. 

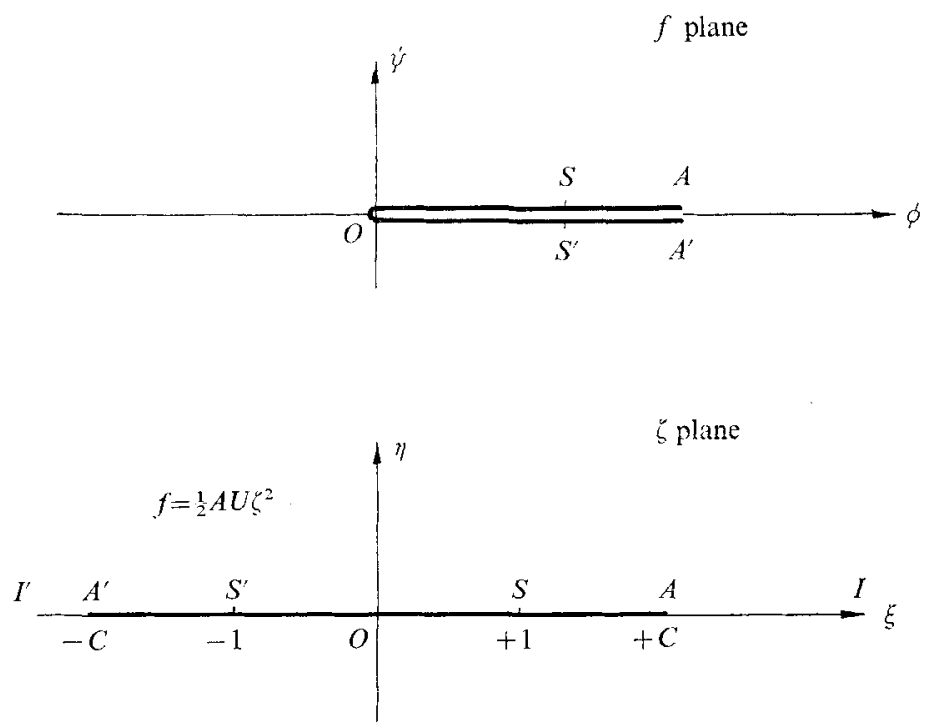

Figure 4. Transformation from the complex potential plane to the $\zeta$ plane.

\section{The problem of the symmetric cavitating plate}

The class of flows under consideration is limited to those flows with an infinite cavity past a plate (see figure 1 ) of frontal width $y_{0}$, total arc-length $s_{0}$, having a continuous slope except at the nose where the vertex angle is $2 \alpha$, with $0 \leqslant \alpha \leqslant \pi$. It is further possible to have a rear stretch of the plate ( $S A$ and $S^{\prime} A^{\prime}$ say) at the cavity pressure $p=p_{c}$ while it remains everywhere wetted; in other words, $S A$ and $S^{\prime} A^{\prime}$ are free streamlines. In addition, we assume that the pressure $p$ on the plate satisfies $p \geqslant p_{c}$.

The last condition $p \geqslant p_{c}$ is an obvious statement of the fact that the vapour pressure (assumed to equal $p_{c}$ ) is the minimum pressure in the flow. The sections $S A$ and $S^{\prime} A^{\prime}$ are included since free streamlines have already been shown to make up part of Lavrentieff's profiles, and similar results are expected for the present problem. It is more convenient than not accounting for this expectation from the beginning. Note, also, that the assumption imposes no undue restrictions on the problem, since the actual locations of $S$ and $S^{\prime}$ are not known a priori, but must be found as part of the optimization requirement.

By proper choice of origin and magnification, the complex potential plane $f=\phi+i \psi$ is mapped to the upper half $\zeta=\xi+i \eta$ plane (see figure 4 ) by

$$
f=\frac{1}{2} A U \zeta^{2}
$$

where $\phi$ is the velocity potential, $\psi$ the stream function, and $A$ a real, positive constant which is chosen so that $S^{\prime} O S$ maps to $|\xi| \leqslant 1$. Tho sections $S A$ and $S^{\prime} A^{\prime}$ map to $1 \leqslant|\xi| \leqslant c$, where $c \geqslant 1$ (equality holds only if $S=A, S^{\prime}=A^{\prime}$ ); the remaining sections of the free streamlines lie on $c \leqslant|\xi| \leqslant \infty$.

We shall adopt the same notation as used by Wu \& Whitney (1972) in part 1 
of this paper (hereafter referred to as part 1). In particular, the complex velocity $w$ and the logarithmic hodograph $w$ are

$$
\begin{gathered}
w=d f / d z=u-i v=q e^{-i \theta} \\
\omega(\zeta)=\log (U / w)=\log (U / q)+i \theta \equiv \tau(\xi, \eta)+i \theta(\xi, \eta) .
\end{gathered}
$$

The pressure $p$ is given by Bernoulli's law

$$
p-p_{c}=\frac{1}{2} \rho\left(U^{2}-q^{2}\right)=\frac{1}{2} \rho U^{2}\left(1-e^{-2 \tau}\right) .
$$

On the free streamlines $S I$ and $S^{\prime} I^{\prime}, p \equiv p_{c}$; therefore, by (4),

$$
\tau(\xi, 0+)=0 \quad(|\xi| \geqslant 1) .
$$

If the boundary value of $\omega$ on $S^{\prime} O S$ is denoted by

$$
\omega(\xi+i 0)=\tau(\xi, 0+)+i \theta(\xi, 0+) \equiv \Gamma(\xi)+i \beta(\xi) \quad(|\xi| \leqslant 1)
$$

then, since $p_{c}$ is the minimum pressure in the flow, the inequality

$$
\Gamma(\xi) \geqslant 0 \quad(|\xi| \leqslant 1)
$$

follows by (4); and, since the pressure is continuous at $S$ and $S^{\prime}$,

$$
\Gamma( \pm 1)=0 \text {. }
$$

The hodograph variable $\omega(\zeta)$ may be split into two parts, $\omega(\zeta)=\omega_{0}(\zeta)+\omega_{1}(\zeta)$, where $\omega_{0}$ accounts for the singular behaviour of $\omega$ at the stagnation point $\zeta=0$, and $\omega_{1}\left(\equiv \Gamma_{1}+i \beta_{1}\right.$, for $\left.|\xi| \leqslant 1\right)$ is analytic in the entire upper half plane including the real axis. It can be shown, in fact, that

$$
\omega(\zeta)=\frac{2 \alpha}{\pi} \log \left\{\left[\left(\zeta^{2}-1\right)^{\frac{1}{2}}+i\right] / \zeta\right\}+\omega_{1}(\zeta)
$$

where $\left(\zeta^{2}-1\right)^{\frac{1}{2}}$ is defined in the $\zeta$ plane cut along the real axis $|\xi| \leqslant 1$ and is positive for $\zeta=\xi>1$; the logarithm function is defined to be that branch which is real for a real, positive argument, with a cut along the negative real axis of the argument. Letting $\zeta \rightarrow \xi+i 0,|\xi| \leqslant 1$, in (9) and comparing this with (6), we have

$$
\begin{gathered}
\Gamma(\xi)=\frac{2 \alpha}{\pi} \log \left\{\left[1+\left(1-\xi^{2}\right)^{\frac{1}{2}}\right] /|\xi|\right\}+\Gamma_{1}(\xi), \\
\beta(\xi)=\alpha \operatorname{sgn} \xi+\beta_{1}(\xi),
\end{gathered}
$$

so that the proper logarithmic singularity of $\Gamma=\operatorname{Re} \omega=\log (U / q)$ and the proper jump in flow angle, $\beta(0+)-\beta(0-)=2 \alpha$, are exhibited at $\zeta=0$. From the conditions required of $\omega$ on the free surface and at infinity, and from our choice for $\omega_{0}$, it readily follows that

$$
\begin{gathered}
\Gamma_{1}(\xi)=\operatorname{Re}\left\{\omega_{1}(\xi+i 0)\right\}=0 \quad(|\xi|>1) \\
\omega_{1}(\zeta) \rightarrow 0 \text { as }|\zeta| \rightarrow \infty .
\end{gathered}
$$

Finally, $\Gamma$ in $(10 a)$ must be chosen so that inequality (7) is satisfied. Also, (8) and $(10 a)$ imply

$$
\Gamma_{1}( \pm 1)=0
$$


The function $\omega_{1}$ is best determined for the so-called inverse problem by regarding either $\Gamma_{1}(\xi)$ or $\beta_{1}(\xi)$ as a known function of $\xi$. This information, together with (11) and (12), determines $\omega_{1}$ uniquely. We now suppose that $\Gamma_{1}=\operatorname{Re} \omega_{1}$ is given which satisfies (13) and is Hölder continuous (see part 1 for the definition) on $|\xi| \leqslant 1$. The Dirichlet problem for $\omega_{1}$,

$$
\operatorname{Re}\left\{\omega_{1}(\xi+i 0)\right\}=\left\{\begin{array}{l}
\Gamma_{1}(\xi) \text { for }|\xi| \leqslant 1, \\
0 \text { for }|\xi|>1,
\end{array}\right\}
$$

together with (12), has the solution

$$
\omega_{1}(\zeta)=-\frac{i}{\pi} \int_{-1}^{1} \frac{\Gamma_{1}(t) d t}{t-\zeta}
$$

which may be verified by letting $\zeta \rightarrow \xi+i 0(|\xi| \leqslant 1)$ in $(15)$ and using Plemelj's formula (e.g. see Muskhelishvili 1953, §17). The imaginary part of $\omega_{1}$ on $S^{\prime} O S$ is found to be

$$
\beta_{1}(\xi)=-\frac{1}{\pi} \oint_{-1}^{1} \frac{\Gamma_{1}(t) d t}{t-\xi} \quad(|\xi| \leqslant 1)
$$

where $\oint$ denotes the Cauchy principal value. The Hölder continuity of $\beta_{1}$ follows from the assumptions already made on $\Gamma_{1}$. (Note that if $\Gamma_{1}$ does not satisfy (13), but approaches a non-zero value at an endpoint, then $\beta_{1}$, as given by (16), will have a logarithmic singularity at that endpoint.) By (2) and (3), the plate shape and the cavity boundary are given parametrically by

$$
z(\xi)=x(\xi)+i y(\xi)=A \int_{0}^{\xi} e^{\omega(\zeta) \zeta} \zeta \xi
$$

and, since the plate is symmetric, its frontal width is given by

$$
y_{0}=\operatorname{Im} A \int_{-c}^{c} e^{\omega(\zeta)} \zeta d \zeta
$$

It is convenient for subsequent analysis to convert this expression for $y_{0}$ to an integral from -1 to +1 . To do this, we first continue $\omega(\zeta)$ into the lower half plane by $\omega(\bar{\zeta})=-\overline{\omega(\zeta)}$. Next, we consider the function $\zeta e^{\omega(\zeta)}$ which appears in the integrand in (18). This function is uniquely determined by the jump in its value (due to the discontinuity of $\omega$ ) across the cut $|\xi| \leqslant 1$ and by its expansion for large $|\zeta|$ (see Muskhelishvili 1953, §78). In fact, it can be shown that

$$
\zeta e^{(\omega(\zeta)}=\frac{1}{\pi i} \int_{-1}^{1} \frac{t e^{i \beta(t)} \sinh \Gamma(t) d t}{t-\zeta}+\frac{i}{\pi} \int_{-1}^{1} \Gamma(t) d t+\zeta
$$

in which the first integral exhibits the correct discontinuity across the cut $|\xi| \leqslant 1$ and the last two terms are required by the expansion of the left side of (19) for $|\zeta| \gg 1$ (see Whitney 1969). In this expansion, we use the integral representation

$$
\omega(\zeta)=-\frac{i}{\pi} \int_{-1}^{1} \frac{\Gamma(t) d t}{t-\zeta}
$$


which is found by exactly the same procedure as that used in determining $\omega_{1}(\zeta)$ (see (14) and (15)). By substituting (19) into (18), and noting that $\beta(-\xi)=-\beta(\xi)$, we obtain

$$
y_{0}=\frac{A}{\pi}\left\{\int_{-1}^{1} t \sinh \Gamma(t)\left[\sin \beta(t)+\cos \beta(t) \log \left(\frac{c-t}{c+t}\right)\right] d t+2 c \int_{-1}^{1} \Gamma(t) d t\right\} .
$$

An element of plate arc-length $d s$ is found from (17), (5), and (6) to be

$$
d s=|d z|=\left\{\begin{array}{l}
A e^{\Gamma(\xi)}|\xi| d \xi \text { for }|\xi| \leqslant 1, \\
A|\xi| d \xi \text { for }|\xi|>1
\end{array}\right.
$$

Thus, the total arc-length of the plate is given by

$$
s_{0}=\int_{-c}^{c} d s=A\left[\left(c^{2}-1\right)+\int_{-1}^{1} e^{\Gamma(t)}|t| d t\right]
$$

The complex force acting on a plate element $d z$ is given by

or, by (4), (5), (6), and (17),

$$
d F=\left(p-p_{c}\right)(-i d z)
$$

$$
d F=-\frac{i}{2} \rho U^{2} A\left(1-e^{-2 \Gamma}\right) e^{\Gamma+i \beta} \xi d \xi \quad(|\xi| \leqslant 1)
$$

and $d F=0$ for $|\xi|>1$. By integrating this expression, noting again the asymmetry of $\beta(\xi)$, we obtain for the drag $D$

$$
D=\rho U^{2} A \int_{-1}^{1} t \sinh \Gamma(t) \sin \beta(t) d t,
$$

and the lift vanishes, as we should expect for a symmetric shape at zero angle of incidence. An alternative expression for the integral which appears in (23), and also in the expression for the width (21), may be obtained by substituting (20) in the left side of (19) and expanding this equation for large $|\zeta|$. By matching the coefficients of $\zeta^{-1}$ on both sides of this expansion, we obtain the identity

$$
\int_{-1}^{1} t \sinh \Gamma(t) \sin \beta(t) d t=\frac{1}{2 \pi}\left(\int_{-1}^{1} \Gamma(t) d t\right)^{2} .
$$

Since $\rho$ and $U$ are kept constant in the minimization of the drag, it is convenient to set $D^{*}=D / \frac{1}{2} \rho U^{2}$ so that $D^{*}$ has the dimension of length. By (23) and (24) we have

$$
D^{*}=\frac{A}{\pi}\left(\int_{-1}^{1} \Gamma(t) d t\right)^{2} .
$$

\section{The minimum drag problem}

In $\$ 2$ we showed that the problem of minimizing the drag of a symmetric profile of given width and arc-length reduces to finding functions $\Gamma_{1}(\xi)$ and $\beta_{1}(\xi)$ and constants $A, c$ and $\alpha$, such that $D^{*}$ in (25) is a minimum subject to the constraints (21) and (22), in which $y_{0}$ and $s_{0}$ are given fixed quantities. The functions $\Gamma(\xi)$ and $\beta(\xi)$ in (21), (22) and (25) are related to $\Gamma_{1}(\xi), \beta_{1}(\xi)$ and the half vertex 
angle of the plate $\alpha$ by $(10)$; furthermore, $\beta_{1}(\xi)$ is related to $\Gamma_{1}(\xi)$ by $(16)$, in which $\Gamma_{1}$ satisfies the end conditions (13) and is chosen so that $\Gamma$ in $(10 \alpha)$ is positive. Equivalently, we may state this problem in terms of $\Gamma(\xi), \beta(\xi), A$, and $c$, and omit further reference to $\Gamma_{1}(\xi), \beta_{1}(\xi)$, and $\alpha$; however, the discontinuous behaviour of $\Gamma$ and $\beta$, as exhibited in (10), should still be recognized. By letting $\zeta \rightarrow \xi+i 0$ $(|\xi| \leqslant 1)$ in $(20)$, we obtain the identity

$$
\beta(\xi)=-\frac{1}{\pi} \oint_{-1}^{1} \frac{\Gamma(t) d t}{t-\xi} \equiv-H_{\xi}[\Gamma(t)]
$$

which may also be verified by (10) and (16). In the above, the symbol $H_{\xi}$ denotes the finite Hilbert transform. Finally, in (21), (22), and (25), the factor $A$ is a real, positive constant and the parameter $c$, which determines the location of the endpoints of the plate in the $\zeta$ plane, satisfies $1 \leqslant c<\infty$.

This problem is equivalent to finding a pair of extremal arcs, $\Gamma(\xi)$ and $\beta(\xi)$, which satisfy (26) and minimize the functional

$I[\Gamma(\xi), \beta(\xi) ; A, c]=D^{*}-\lambda_{1} s_{0}-2 \pi \lambda_{2} y_{0}$

$$
=A \int_{-1}^{1} f\left(\Gamma(t), \beta(t), t ; \lambda_{1}, \lambda_{2}, c\right) d t+\frac{A}{\pi}\left(1-\lambda_{2}\right)\left(\int_{-1}^{1} \Gamma(t) d t\right)^{2},
$$

where, by (21), (22), (24) and (25),

$f\left(\Gamma(\xi), \beta(\xi), \xi ; \lambda_{1}, \lambda_{2}, c\right)=-\lambda_{1}\left[\frac{1}{2}\left(c^{2}-1\right)+e^{\Gamma(\xi)|\xi|]}\right.$

$$
-2 \lambda_{2}[\xi \sinh \Gamma(\xi) \cos \beta(\xi) \log \{(c-\xi) /(c+\xi)\}+2 c \Gamma(\xi)]
$$

In the above, $\lambda_{1}, \lambda_{2}$ are Lagrange multipliers, and the integral identity (24) has been used in the expression (21) for $y_{0}$.

The general variational problem of this type has been investigated earlier by Wu \& Whitney $(1971,1972)$. For the present problem the method of solution will follow the same approach with some slight modifications. Let the set $\{\Gamma(\xi), \beta(\xi), A, c\}$ denote the optimal solution and let $\{\widetilde{\Gamma}(\xi), \tilde{\beta}(\xi), \tilde{A}, \tilde{c}\}$ be an arbitrary neighbouring set which also satisfy (21), (22), and (26). The differences $\delta \Gamma(\xi)=\widetilde{\Gamma}-\Gamma, \delta \beta(\xi)=\widetilde{\beta}-\beta, \delta A=\tilde{A}-A, \delta c=\tilde{c}-c$, form a set of small variations; $\delta A, \delta_{c}$, and either $\delta \Gamma$ or $\delta \beta$ are independent of one another since by (26), $\delta \Gamma$ and $\delta \beta$ are related by

$$
\delta \beta(\xi)=-H_{\xi}[\delta \Gamma(t)]
$$

The variation of the functional $I$, about its optimal value, due to the variations $\{\delta \Gamma, \delta \beta, \delta A, \delta c\}$ is given by

$$
\begin{aligned}
\Delta I & =I[\Gamma+\delta \Gamma, \beta+\delta \beta ; A+\delta A, c+\delta c]-I[\Gamma, \beta ; A, c] \\
& =\delta I+\frac{1}{2 !} \delta^{2} I+\ldots,
\end{aligned}
$$

where, by (27), the first variation $\delta I$ and second variation $\delta^{2} I$ are

$$
\begin{gathered}
\delta I=I_{A} \delta A+I_{c} \delta c+A \int_{-1}^{1}\left\{\left[f_{\Gamma}+\frac{2}{\pi}\left(1-\lambda_{2}\right) J\right] \delta \Gamma(t)+f_{\beta} \delta \beta(t)\right\} d t \\
\delta^{2} I=2 I_{A c} \delta A \delta c+I_{c c}(\delta c)^{2}+2 \delta A \int_{-1}^{1}\left\{\left[f_{\Gamma}+\frac{2}{\pi}\left(1-\lambda_{2}\right) J\right] \delta \Gamma+f_{\beta} \delta \beta\right\} d t \\
+A \int_{-1}^{1}\left\{f_{\Gamma \Gamma}(\delta \Gamma)^{2}+2 f_{\Gamma \beta} \delta \Gamma \delta \beta+f_{\beta \beta}(\delta \beta)^{2}\right\} d t+\frac{2 A}{\pi}\left(1-\lambda_{2}\right)(\delta J)^{2},
\end{gathered}
$$


where the subscripts denote partial differentiation, and

$$
J=\int_{-1}^{1} \Gamma(t) d t, \quad \delta J=\int_{-1}^{1} \delta \Gamma(t) d t .
$$

For $I$ to be a minimum, we must have $\delta I=0$ and $\delta^{2} I>0$ for arbitrary $\delta A, \delta c$, and $\delta \Gamma(\xi)$. Therefore, the three terms in the expression (29) for $\delta I$ must vanish separately to ensure $\delta I=0$. The first of these requires $I_{A}=\partial I / \partial A=I / A=0$, or

$$
\int_{-1}^{1} f\left(\Gamma(t), \beta(t), t ; \lambda_{1}, \lambda_{2}, c\right) d t+\frac{1}{\pi}\left(1-\lambda_{2}\right) J^{2}=0,
$$

or, by $(27 a)$ and (31),

$$
D^{*}=\lambda_{1} s_{0}+2 \pi \lambda_{2} y_{0}
$$

The second equation comes from $\partial I / \partial c=0$, or by $(27 a, b)$,

$$
\frac{1}{2} \int_{-1}^{1} f_{c} d t=\lambda_{1} c-2 \lambda_{2} \int_{-1}^{1}\left\{\frac{t^{2} \sinh \Gamma(t) \cos \beta(t)}{c^{2}-t^{2}}+\Gamma(t)\right\} d t=0 .
$$

Finally, for the last integral of (29), we substitute (28) for $\delta \beta$, then change the order of integration, giving

$$
\int_{-1}^{1}\left\{f_{\Gamma}+\frac{2}{\pi}\left(1-\lambda_{2}\right) J+H_{\xi}\left[f_{\beta}\right]\right\} \delta \Gamma(t) d t=0 .
$$

Now, since $\delta \Gamma(\xi)$ is arbitrary, we obtain the nonlinear singular integral equation

$$
f_{\Gamma}\left(\Gamma, \beta, \xi ; \lambda_{1}, \lambda_{2}, c\right)+H_{\xi}\left[f_{\beta}\left(\Gamma, \beta, t ; \lambda_{1}, \lambda_{2}, c\right)\right]=-\frac{2}{\pi}\left(1-\lambda_{2}\right) \int \Gamma(t) d t,
$$

where, by $(27 b)$,

$$
\left.\begin{array}{l}
f_{\Gamma}=-\lambda_{1} e^{\Gamma(\xi) \mid \xi}-2 \lambda_{2}\left[\xi \cosh \Gamma(\xi) \cos \beta(\xi) \log \left(\frac{c-\xi}{c+\xi}\right)+2 c\right], \\
f_{\beta}=2 \lambda_{2} \xi \sinh \Gamma(\xi) \sin \beta(\xi) \log \left(\frac{c-\xi}{c+\xi}\right) .
\end{array}\right\}
$$

This integral equation, which contains $\lambda_{1}, \lambda_{2}$, and $c$ as parameters, is to be solved together with the linear integral equation (26) for the extremal arcs $\Gamma(\xi)$ and $\beta(\xi)$. The Lagrange multipliers $\lambda_{1}, \lambda_{2}$ can be determined from (32) and (33). The parameter $c$ is most conveniently determined from the relation

$$
s_{0} / y_{0}=k
$$

say, where $k$ is a specified quantity, and $y_{0}, s_{0}$ are given by (20), (21). Finally, the optimal drag coefficient (based on plate width) is given by

$$
C_{D}=D / \frac{1}{2} \rho U^{2} y_{0}=D^{*} / y_{0}
$$

in which $D^{*}$ assumes the form (25). (Note that actual calculation of the parameter $A$ is curtailed as $A$ drops out of the expressions for $C_{D}$ and $s_{0} / y_{0}$. It is necessary, however, to consider the variation $\delta A$ in order to obtain (32), which relates $s_{0} / y_{0}$ and $C_{D}$ )

As explained previously, this optimal drag coefficient is a minimum if the second variation $\delta^{2} I>0$. Upon using (24) to derive an alternative expression for the second variation of $J^{2}$, the last two terms on the right-hand side of $(30)$ 
become of the same form and can be readily combined. Further noting that $I_{c}=0$ implies automatically $I_{A c}=0$, since $I_{A c}=\partial I_{c} / \partial A=I_{c} / A$, the inequality $\delta^{2} I>0$ now reduces to

$$
A(\delta c)^{2} \int f_{c c} d t+A \int\left\{g_{\Gamma \Gamma}(\delta \Gamma)^{2}+2 g_{\Gamma \beta} \delta \Gamma \delta \beta+g_{\beta \beta}(\delta \beta)^{2}\right\} d t>0
$$

where $g \equiv f+2 A\left(1-\lambda_{2}\right) \xi \sinh \Gamma(\xi) \sin \beta(\xi)$. Since $A>0$, this inequality holds if both integrals are positive. The first term is positive when, by (27),

$$
\frac{1}{2} \int_{-1}^{1} f_{c c} d t=-\lambda_{1}+2 \lambda_{2} c \int_{-1}^{1} \frac{t^{2} \sinh \Gamma(t) \cos \beta(t) d t}{\left(c^{2}-t^{2}\right)^{2}}>0 .
$$

It has been shown by $W u \&$ Whitney $(1971,1972)$ that a necessary condition for the second integral in (38) to be positive is that

$$
g_{\Gamma \Gamma}+g_{\beta \beta}=-\lambda_{1} e^{\Gamma(\xi)}|\xi|>0 \quad(|\xi|<1)
$$

or simply

$$
\lambda_{1}<0 \text {, }
$$

since $\Gamma$ is real. Once an optimal solution is found, conditions (39) and (40) may be checked to determine if the solution is actually a minimum.

The singular integral equation (35) can be reduced to an integral equation with a regular kernel by using the identity

$$
\xi \cosh \Gamma(\xi) \cos \beta(\xi)=H_{\xi}[t \sinh \Gamma(t) \sin \beta(t)]+\xi,
$$

which can be obtained by letting $\zeta \rightarrow \xi \pm i 0(|\xi| \leqslant 1)$ in (19). Finally, substitution of $\xi \cosh \Gamma \cos \beta$ given by (41) into (35) yields

where

$$
\begin{gathered}
2 \lambda_{2} \int_{-1}^{1} t \sinh \Gamma(t) \sin \beta(t) K(t, \xi ; c) d t-\lambda_{1} e^{\Gamma(\xi)}|\xi| \\
=2 \lambda_{2}\left(2 c+\xi \log \{(c-\xi) /(c+\xi)\}-\frac{2}{\pi}\left(1-\lambda_{2}\right) \int_{-1}^{1} \Gamma(t) d t\right. \\
K(t, \xi ; c)=\frac{1}{\pi} \log \{(c-t)(c+\xi) /(c+t)(c-\xi)\} /(t-\xi)
\end{gathered}
$$

We note that the kernel $K(t, \xi ; c)$ is regular even at $t=\xi$.

It is now possible to show that, if an optimal shape exists, it must have a flat nose, i.e. $\alpha=\frac{1}{2} \pi$. To show this, we first note that by $(42) e^{\Gamma(\xi)}|\xi|$ possesses a regular series expansion about $\xi=0$ of the form

$$
e^{\Gamma(\xi)}|\xi|=c_{0}+c_{1} \xi^{2}+\ldots
$$

where the explicit forms of $c_{0}, c_{1}, \ldots$, etc., are easily found but are unnecessary. On the other hand, from $(10 \alpha)$ we can deduce that

$$
\begin{aligned}
e^{\Gamma(\xi)|\xi|} & =\left[\left\{1+\left(1-\xi^{2}\right)^{\frac{1}{2}}\right\} /|\xi|\right]^{2 \alpha / \pi}|\xi| e^{\Gamma_{1}(\xi)} \\
& =(2)^{2 \alpha i \pi}|\xi|^{1-2 \alpha \mid \pi} e^{\Gamma_{1}(0)}+O\left(|\xi|^{2(1-\alpha \mid \pi)}\right)
\end{aligned}
$$

as $|\xi| \rightarrow 0$, since $\Gamma_{\mathbf{1}}(\xi)$ is assumed to be continuous, at $\xi=0$, in particular. This expansion agrees with (43) if and only if $\alpha=\frac{1}{2} \pi$, i.e. when the nose of the plate is flat.

Since there are no known analytical methods for solving the system of integral equations, (26), (42), our only recourse is in some numerical methods. These 
attempts have not been successful; however, in order to illustrate some of the many difficulties which beset such procedures, we briefly mention one of the schemes that has been tried. First, the integrals in (26), (32), (33) and (42) were approximated by numerical quadratures which involved the values of $\Gamma(\xi)$ and $\beta(\xi)$ at $N$ points $\left\{\xi_{i}\right\}$ from -1 to +1 . An initial guess of $\left\{\Gamma\left(\xi_{i}\right)\right\}$ was made and the set $\left\{\beta\left(\xi_{i}\right)\right\}$ was then calculated by (26). Next the Lagrange multipliers $\lambda_{1}, \lambda_{2}$ were found from (32) and (33), in which the current values of $\Gamma$ and $\beta$ were used. Finally, new values of $\left\{\Gamma\left(\xi_{i}\right)\right\}$ were calculated by solving for $\Gamma(\xi)$ in $(42)$, and the process was repeated, with the hope that the iterations would converge. These calculations were done for arbitrary values of $c \geqslant 1$, with different $c$ 's corresponding to different ratios $s_{0} / y_{0}$.

As mentioned above, this method and several similar ones did not work. Among the more disagreeable features encountered are the following: (i) the iterations do not always converge; (ii) the values of $\Gamma\left(\xi_{i}\right)$, as given by the solution of (42) for $\Gamma(\xi)$, are not always positive, so that (7) is violated; and (iii) the values of $\Gamma$ at $\xi= \pm 1$ is not zero, so that $\beta$ in (26) becomes large as $\xi \rightarrow \pm 1$ and integration of terms involving $\sin \beta$ and $\cos \beta$ by numerical quadrature fails. Corrective steps were taken, such as enforcing $\Gamma( \pm 1)=0$ at each iteration step, and by increasing $N$; these measures did not help, however.

Note that $\beta(\xi)$ in (26) has a logarithmic singularity at $\xi= \pm 1$ if

$$
\Gamma( \pm 1)=\text { const. } \neq 0,
$$

and $\beta$ has a higher-order (branch-type) singularity if $\Gamma$ itself is singular at the endpoints (e.g. see Muskhelishvili 1953, §29). By studying the linear case, in which the functionals are quadratic in $\Gamma$ and $\beta$ (see Wu \& Whitney 1971, 1972), it was deduced that the endpoint condition (7) cannot be satisfied in general. If this conclusion is also true for the present nonlinear case, it is likely that a solution to (26) and (42) will have no direct physical relevance since $\Gamma$ and $\beta$ will be singular at $\xi= \pm 1$. Nevertheless, such a solution (if one exists) would provide a mathematical bound for the drag which could then be used in judging the 'degree of optimality' of results obtained by other (approximate) methods, such as that presented in $\$ 4$.

\section{Solution by expansions in finite Fourier series}

We now investigate a method to obtain an approximate optimal solution by the expanding $\Gamma_{1}(\xi)$ and $\beta_{1}(\xi)$ in Fourier series in which the constant coefficients are chosen so that the drag is minimized, subject to the same isoperimetric constraints as before. Let the expansion for $\Gamma_{1}$ be given by

$$
\Gamma_{1}(\xi)=-\sum_{n=1}^{N} a_{n} \sin (2 n-1) \theta,
$$

where $\xi=\cos \theta(0 \leqslant \theta \leqslant \pi)$. This $\Gamma_{1}$ satisfies (13) and the required symmetry property. From the identity

$$
\oint_{0}^{\pi} \frac{\sin m \phi \sin \phi d \phi}{\cos \phi-\cos \theta}=-\pi \cos m \theta
$$


we see that (16) is satisfied, term by term, if

$$
\beta_{1}(\xi)=-\sum_{n=1}^{N} a_{n} \cos (2 n-1) \theta
$$

Upon setting $\alpha=\frac{1}{2} \pi$ in (9) (as dictated by the result of $\S 3$ ), we obtain

$$
\omega(\zeta)=\log \left\{\left[\left(\zeta^{2}-1\right)^{\frac{1}{2}}+i\right] / \zeta\right\}+\omega_{1}(\zeta)
$$

Therefore, by (17), the frontal width of the plate is given by

$$
y_{0}=-2 A \operatorname{Im} \int_{0}^{-c} e^{\omega_{1}(\zeta)}\left[\left(\zeta^{2}-1\right)^{\frac{1}{2}}+i\right] d \zeta
$$

This integral is most easily evaluated by the change of variables

$$
\zeta=\frac{1}{2}\left(\nu+\nu^{-1}\right)
$$

which maps the upper half $\zeta$ plane into the half circle $|\nu| \leqslant 1, \operatorname{Im} \nu \geqslant 0$ (see figures 4 and 5) and maps the endpoints of the plate $\zeta= \pm c$ to $\nu=\mp \kappa$, where

$$
\kappa=c-\left(c^{2}-1\right)^{\frac{1}{2}}
$$

It is readily verified that $\omega_{1}$ as a function of $\nu$ is given by

$$
\omega_{1}(\nu)=i \sum_{n=1}^{N} a_{n} \nu^{2 n-1} \equiv i \Omega(\nu)
$$

From (46) to (49), the expression for the width now becomes

$$
y_{0}=\frac{A}{2} \operatorname{Im} \int_{i}^{\kappa} e^{i \Omega(\nu)}\left(\nu+2 i-2 \nu^{-1}-2 i \nu^{-2}+\nu^{-3}\right) d \nu
$$

which is evaluated by taking the path of integration $L_{\varepsilon}$ shown in figure 5 . In the limit $\epsilon \rightarrow 0$, it can be shown (see Whitney 1969) that

$$
\begin{array}{r}
y_{0}=\frac{A}{4}\left\{\int_{0}^{\kappa}\left[\left(2 t-\frac{\left\{2-\Omega^{\prime}(t)\right\}^{2}}{t}\right) \sin \Omega(t)+\left(4+\frac{\Omega^{\prime \prime}(t)}{t}\right) \cos \Omega(t)\right] d t\right. \\
\left.+\pi\left(2-a_{1}\right)^{2}+\frac{4-\Omega^{\prime}(\kappa)}{\kappa} \cos \Omega(\kappa)-\frac{1}{\kappa^{2}} \sin \Omega(\kappa)\right\}
\end{array}
$$

where $\Omega^{\prime}(t)=d \Omega / d t, \Omega^{\prime \prime}(t)=d^{2} \Omega / d t^{2}$. By $(10 a),(44)$, and (48), the expression for the arc-length (22) becomes

$$
s_{0}=A\left[\frac{1}{4}\left(\kappa+\kappa^{-1}\right)^{2}-1+\int_{0}^{\pi} \exp \left\{-\sum_{n=1}^{N} a_{n} \sin (2 n-1) \theta\right\}(1+\sin \theta) \sin \theta d \theta\right] .
$$

Finally, from (10a), (25), and (44), the drag is found to depend only on the first of the Fourier coefficients,

$$
D^{*}=\frac{1}{4} A \pi\left(2-a_{1}\right)^{2} \text {. }
$$

The optimization problem reduces to minimizing $D^{*}$ in (52), subject to the constraints (50) and (51), over the $(N+2)$-dimensional space $\left(A, \kappa, a_{1}, a_{2}, \ldots, a_{N}\right)$. For general values of $N>1$, this problem must be done numerically; however, if $N=1$ the integrals in (50) and (51) may be evaluated in terms of special 


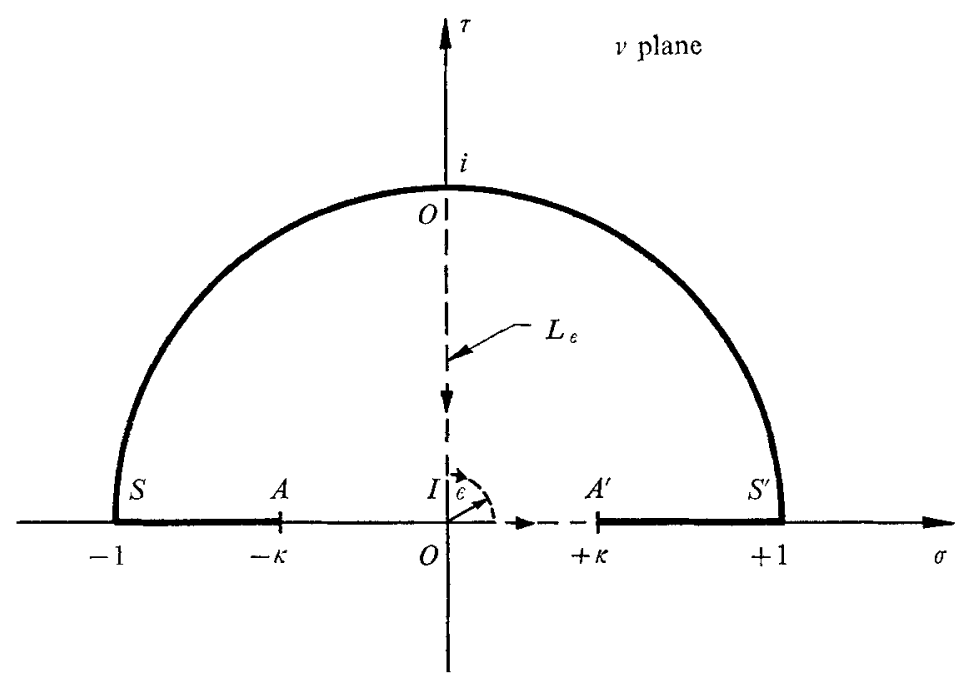

FIGURe 5. The path of integration $L_{\varepsilon}$ in the $v$ plane from $\nu=i$ to $\nu=\kappa$.

$$
\zeta=-\frac{1}{2}(\nu+1 / \nu)
$$

functions. Note that by $(10 b)$ and $(45), \beta(\xi)=\frac{1}{2} \pi \operatorname{sgn} \xi-a_{1} \cos \theta$, for the case $N=1$, so the plate section $S^{\prime} O S$ is convex (or concave) when viewed from the approaching flow as $a_{1}$ is positive (or negative). This section is a flat plate, corresponding to the Lavrentieff profile (see $\S 1$ ) when $a_{1}=0$.

With $N=1$ in (49)-(51), $\Omega(t)=a_{1} t$, so that (50) and (51) can be reduced to

$$
\begin{gathered}
y_{0}=\frac{1}{4} A\left\{\left(2 a_{1}^{-2}+4 a_{1}^{-1}-\kappa^{-2}\right) \sin \left(a_{1} \kappa\right)+\left(4 \kappa^{-1}-a_{1} \kappa^{-1}-2 \kappa a_{1}^{-1}\right) \cos \left(a_{1} \kappa\right)\right. \\
\left.+\left(2-a_{1}\right)^{2}\left[\frac{1}{2} \pi-S i\left(a_{1} \kappa\right)\right]\right\}, \\
s_{0}=A\left[\frac{1}{4}\left(\kappa+\kappa^{-1}\right)^{2}-1+\int_{0}^{\pi} e^{-a_{1} \sin \theta}(1+\sin \theta) \sin \theta d \theta\right] \\
=\frac{1}{4} A\left[\kappa^{2}+\kappa^{-2}+6+4 \pi\left(1+a_{1}^{-1}\right)\left\{L_{1}\left(a_{1}\right)-I_{1}\left(a_{1}\right)\right\}-4 \pi\left\{L_{0}\left(a_{1}\right)-I_{0}\left(a_{1}\right)\right\}\right],
\end{gathered}
$$

where $S i(x)$ is the sine integral, $L_{i}(x)$ and $I_{i}(x)$ are the modified Struve and Bessel functions, respectively (see e.g. Abramowitz \& Stegun 1964).

The problem of finding the optimal plate shape from the class of plates with $N=1$ is now equivalent to extremizing

$$
I\left(A, \kappa, a_{1}\right)=D^{*}\left(A, a_{1}\right)-\lambda_{1} s_{0}\left(A, \kappa, a_{1}\right)-2 \pi \lambda_{2} y_{0}\left(A, \kappa, a_{1}\right),
$$

where, as before, $\lambda_{1}$ and $\lambda_{2}$ are unknown Lagrange multipliers and $D^{*}, s_{0}$ and $y_{0}$, are given by (52), (54) and (53), respectively. If $I$ is extremal, the three partial derivatives $I_{A}, I_{k}$ and $I_{a_{1}}$ must vanish. This gives three relations among the quantities $A, \kappa, a_{1}, \lambda_{1}$ and $\lambda_{2}$. By eliminating $\lambda_{1}$ and $\lambda_{2}$ from these three equations we obtain

$$
D_{A}^{*}\left[s_{0 a_{1}} y_{0 \kappa}-s_{0 \kappa} y_{0 a_{1}}\right]-D_{a_{1}}^{*}\left[s_{0 A} y_{0 \kappa}-s_{0 \kappa} y_{0 A}\right]=0,
$$

where $s_{0 \kappa} \equiv \partial s_{0} / \partial \kappa$, etc. Let the solution of (55) be denoted by $a_{1}=f(\kappa)$. For $\kappa \sim 1(c \sim 1)$ it can be shown, by expansions of (52), (53), (54) and (55), that

$$
a_{1}=f(\kappa)=\{8 /(3 \pi+16)\}(1-\kappa)^{2}-\left\{24 \pi /(3 \pi+16)^{2}\right\}(1-\kappa)^{3}+O(1-\kappa)^{4} .
$$




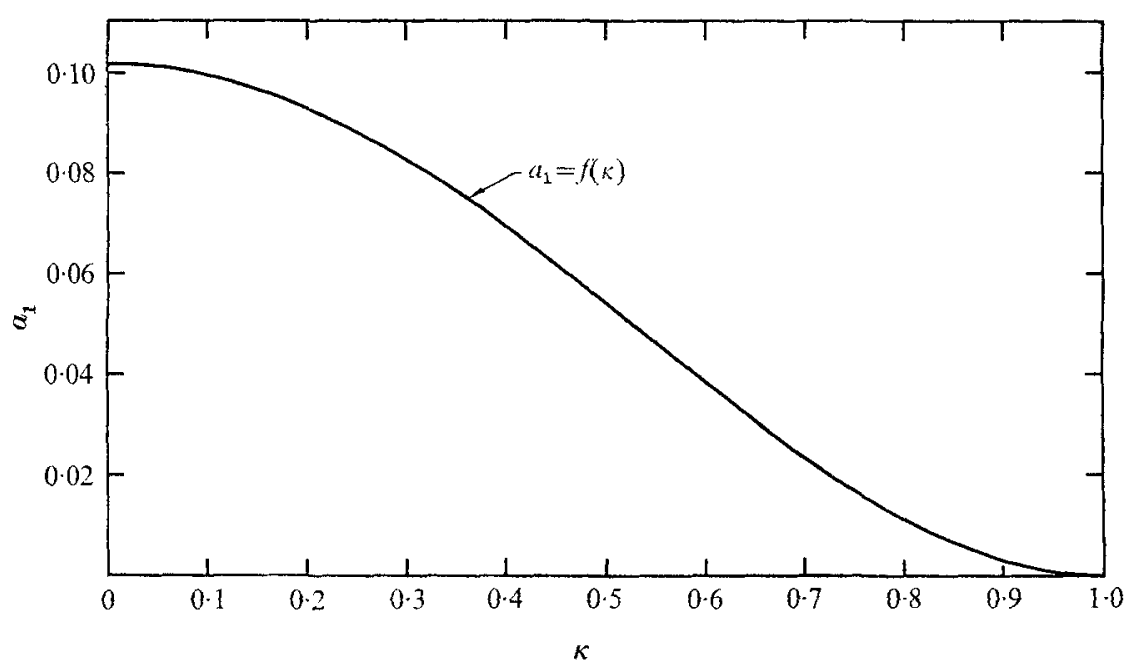

Frgure 6. The curve $a_{1}=f(\kappa)$ satisfying equation (55).

The general solution, as plotted in figure 6 , if found by fixing $\kappa$ at various values between 0 and 1 and numerically solving for $a_{1}$ from (55). As $\kappa \rightarrow 0(c \rightarrow \infty), a_{1}$ is found to be the root of the transcendental equation

$$
a_{1}\left\{L_{0}\left(a_{1}\right)-I_{0}\left(a_{1}\right)\right\}-\left(a_{1}+2\right)\left\{L_{1}\left(a_{1}\right)-I_{1}\left(a_{1}\right)\right\}=\frac{a_{1}^{2}\left(3-2 a_{1}\right)}{\pi\left(4-a_{1}^{2}\right)} .
$$

This root is given by $a_{1} \sim 0.1020$, which provides an upper bound for $a_{1}$; the corresponding optimal shapes are only slightly curved over $S^{\prime} O S$.

This one relation, $a_{1}=f(\kappa)$, is all that is needed to complete the solution, since the factor $A$ drops out of the expressions for the drag coefficient and the ratio $s_{0} / y_{0}$ of are-length to chord. Thus, by (36) and (37)

$$
\begin{gathered}
C_{D}=C_{D}\left(a_{1}, \kappa\right)=D^{*} / y_{0}, \\
k=k\left(a_{1}, \kappa\right)=s_{0} / y_{0} .
\end{gathered}
$$

Evaluation of (57) and (58) (in which $D^{*}, y_{0}$, and $s_{0}$ are given by (52), (53), and (54)) for $a_{1}=f(\kappa)$ gives a parametric representation of $C_{D}$ against $k$. This is plotted in figure 7 , where $C_{D}=2 \pi /(\pi+4)$ is the drag coefficient of a flat plate in infinity cavity flow (see e.g. Lamb 1932). As $k \rightarrow \infty$, it can be shown from (57) and (58) that $C_{D} \sim(4+\pi) /(\pi+8 k)$.

The minimum drag profiles for various values of $k$ are obtained by numerically integrating $d z$ in (17) and, as shown in figure 8, are seen to be quite similar to Lavrentieff's profiles discussed earlier in $\S 1$. However, by expanding $y_{0}$ in (53) and $s_{0}$ in (54) for small $a_{1}$, it can be shown that for $(k-1) \ll 1$

$$
C_{D}=\frac{2 \pi}{4+\pi}\left\{1-\gamma(k-1)^{\frac{1}{2}}+O(k-1)\right\}
$$

where

$$
\gamma=4\{2(\pi+4)\}^{-\frac{1}{2}} \sim 1 \cdot 0584
$$

for the Lavrentieff profiles $\left(a_{1}=0\right)$, and

$$
\gamma=4\left\{\frac{1}{4}(9 \pi+64) /(\pi+4)(3 \pi+16)\right\}^{\frac{1}{2}} \sim 1 \cdot 1641
$$


Theory of optimum shapes in free-surface flows. Part 2

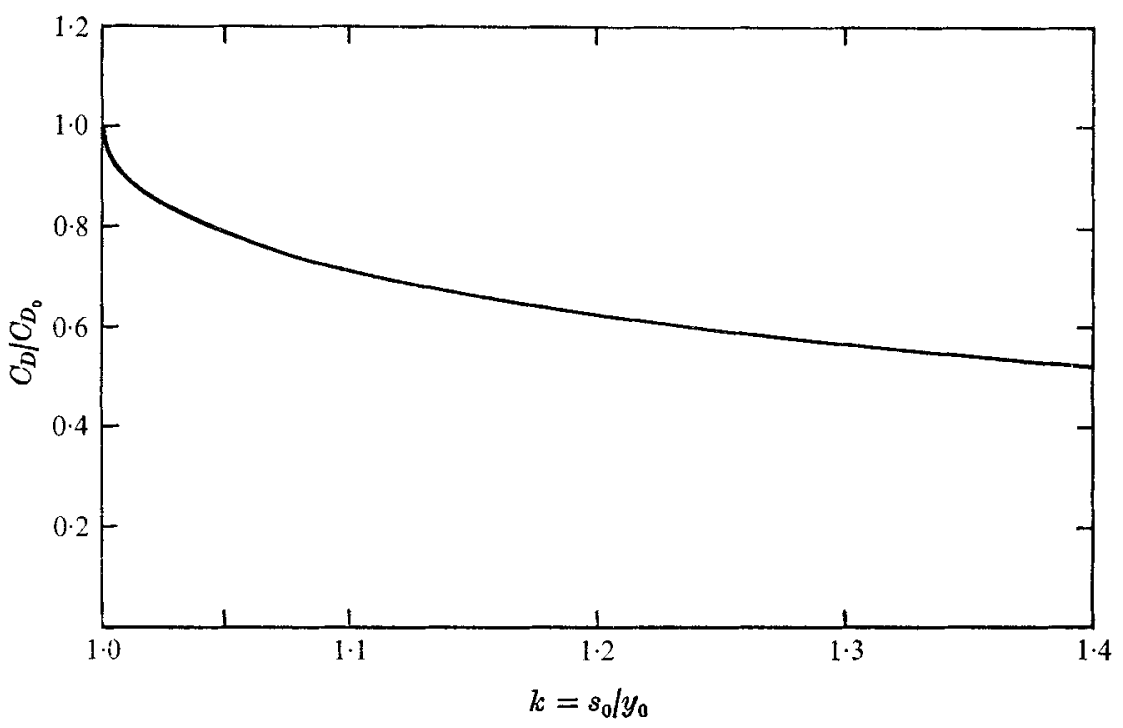

Figure 7. Minimum drag coefficient against $k$ for the case $N=1$. $C_{D} / C_{D_{0}} \sim(4+\pi) /(\pi+8 k)$ as $k \rightarrow 0$.

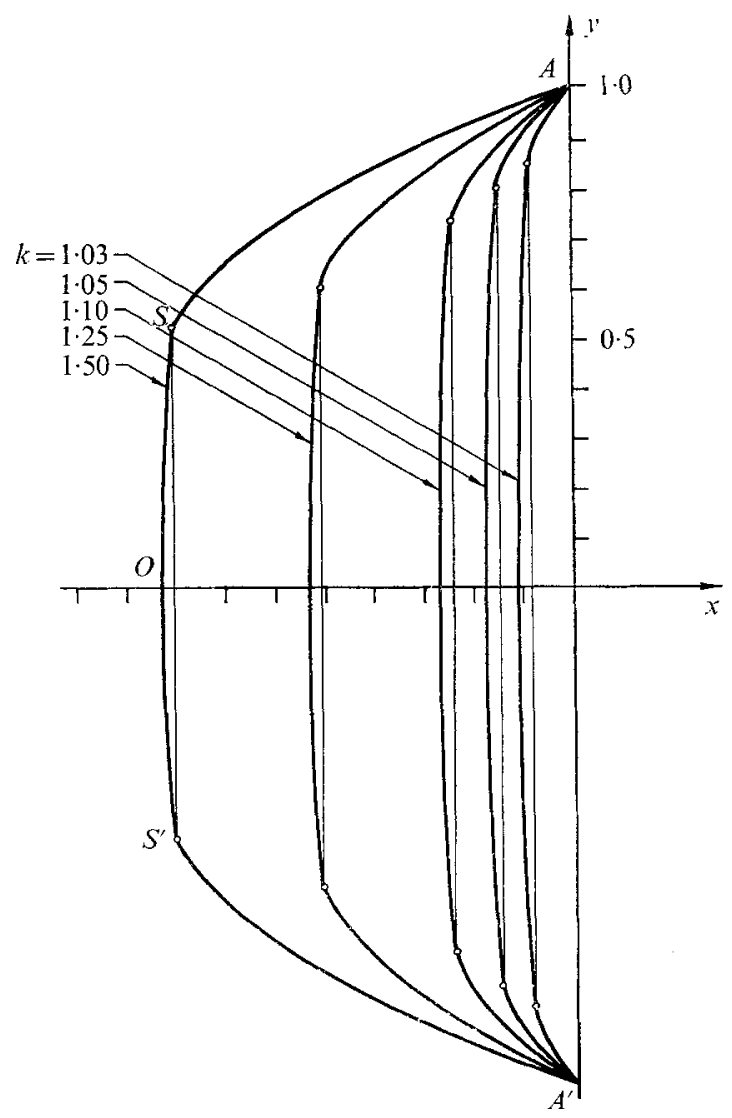

Figure 8. Some optimum plate profiles for the case $N=1$. 
for the profiles in figure 7 . Therefore, for $k$ close to unity, the drag coefficients of the profiles in figure 7 are slightly less than those for Lavrentieff's profiles. This is not too surprising, however, since the constraining conditions in the present problem differ from Lavrentieff's.

The cases $N=2,3, \ldots$, could, in principle, be carried out along similar lines and should result in improved drag coefficients for a given $k=s_{0} / y_{0}$. The numerical examples given by Wu \& Whitney (1971), in which the exact solutions to the variational problems are known, indicate that expansion in Fourier series is a very effective method, at least for the case of quadratic functionals. Whether the same holds true for the present problem, in which the functional is of a different type, remains to be seen.

This paper is based on part of the author's doctoral research which was supported by the National Science Foundation and carried out at the California Institute of Technology under Professor T. Y. Wu, whose interest and encouragement is gratefully acknowledged. The present work was sponsored by the Naval Ship System Command General Hydrodynamics Research and Development Center and the Office of Naval Research, under contract 220(51).

\section{REFERENCES}

ABrayowitz, M. \& Stegun, I. 1964 Handbook of Mathematical Functions. National Bureau of Standards.

Gilbarg, D. 1960 Jets and Cavities. Handbuch der Physik, vol. 9. Springer.

LaMrs, H. 1932 Hydrodynamics (6th edn.). Cambridge University Press.

LAVRENTIEFF, M. 1938 Sur certaines properiétés des fonctions univalentes et leurs applications à la théories des sillages. Mat. Sbornik, 46, 391.

Muskheusshvilt, N. 1953 Singular Integral Equations. Groningen, Holland: Nordhoff.

Whitney, A. K. 1969 Minimum drag profiles in infinite cavity flows. Ph.D. thesis, California Institute of Technology, Pasadena, California.

Wu, T. Y. \& Whitney, A. K. 1971 Theory of optimum shapes in free-surface flows. Calif. Inst. Tech. Rep. E 132 F. 1.

Wu, T. Y. \& WhITNEx, A. K. 1972 Theory of optimum shapes in free-surface flows. Part 1. Optimum profile of sprayless planing surface. J. Fluid Mech., 55, 439. 